Portland State University

PDXScholar

\title{
A Bayesian Hierarchical Approach to Multivariate Nonstationary Hydrologic Frequency Analysis
}

\author{
C. Bracken
}

Bonneville Power Administration, Portland, OR, USA,

K. D. Holman

Bureau of Reclamation, Technical Service Center, Denver, CO

B. Rajagopalan

Department of Civil, Environmental, and Architectural Engineering, University of Colorado at Boulder, Boulder, CO

Hamid Moradkhani

Portland State University, hamidm@pdx.edu

Follow this and additional works at: https://pdxscholar.library.pdx.edu/cengin_fac

Part of the Hydrology Commons

Let us know how access to this document benefits you.

\section{Citation Details}

Bracken, C., Holman, K. D., Rajagopalan, B., \& Moradkhani, H. (2018). A bayesian hierarchical approach to multivariate nonstationary hydrologic frequency analysis. Water Resources Research, 54(1), 243-255.

This Article is brought to you for free and open access. It has been accepted for inclusion in Civil and Environmental Engineering Faculty Publications and Presentations by an authorized administrator of PDXScholar. Please contact us if we can make this document more accessible: pdxscholar@pdx.edu. 


\section{Water Resources Research}

\section{RESEARCH ARTICLE \\ 10.1002/2017WR020403 \\ A Bayesian Hierarchical Approach to Multivariate Nonstationary Hydrologic Frequency Analysis}

Key Points:

- A model for nonstationary multivariate hydrologic frequency analysis is developed

- The model allows for the incorporation of climate covariates and the specification of a nonlinear dependence between variables

- Multivariate frequency analysis capture the dependence between multiple hydrologic variables

Correspondence to:

C. Bracken,

cameron.bracken@gmail.com

Citation:

Bracken, C., Holman, K. D., Rajagopalan, B., \& Moradkhani, H. (2018). A Bayesian hierarchical approach to multivariate nonstationary hydrologic frequency analysis. Water Resources Research, 54, 243-255. https://doi.org/10.1002/ 2017WR020403

Received 13 JAN 2017 Accepted 23 DEC 2017 Accepted article online 4 JAN 2018 Published online 19 JAN 2018

\author{
C. Bracken ${ }^{1}$ (D) K. D. Holman2 ${ }^{(D)}$, B. Rajagopalan 3,4 (D), and H. Moradkhani5 \\ ${ }^{1}$ Bonneville Power Administration, Portland, OR, USA, ${ }^{2}$ Bureau of Reclamation, Technical Service Center, Denver, CO, USA, \\ ${ }^{3}$ Department of Civil, Environmental, and Architectural Engineering, University of Colorado at Boulder, Boulder, CO, USA, \\ ${ }^{4}$ Cooperative Institute for Research in Environmental Sciences, University of Colorado at Boulder, Boulder, CO, USA, \\ ${ }^{5}$ Department of Civil and Environmental Engineering, Portland State University, Portland, OR, USA
}

\begin{abstract}
We present a general Bayesian hierarchical framework for conducting nonstationary frequency analysis of multiple hydrologic variables. In this, annual maxima from each variable are assumed to follow a generalized extreme value (GEV) distribution in which the location parameter is allowed to vary in time. A Gaussian elliptical copula is used to model the joint distribution of all variables. We demonstrate the utility of this framework with a joint frequency analysis model of annual peak snow water equivalent (SWE), annual peak flow, and annual peak reservoir elevation at Taylor Park dam in Colorado, USA. Indices of largescale climate drivers-El Niño Southern Oscillation (ENSO), Pacific Decadal Oscillation (PDO), and Atlantic Multidecadal Oscillation (AMO) are used as covariates to model temporal nonstationarity. The Bayesian framework provides the posterior distribution of the model parameters and consequently the return levels. Results show that performing a multivariate joint frequency analysis reduces the uncertainty in return level estimates and better captures multivariate dependence compared to an independent model.
\end{abstract}

Plain Language Summary In this study, we develop a method for determining the probability of occurrence of rare hydrologic events (e.g., floods). Utilizing modern statistical methods, we are able to estimate occurrence probabilities for multiple hydrologic variables simultaneously while incorporating climate information that changes in time. We apply this technique to estimate occurrence probabilities for streamflow, reservoir elevation, and snow levels for the Taylor Park reservoir in Colorado, USA. This method provides several benefits over traditional methods including reduction of uncertainty and a flexible model structure which allows for the incorporation of climate information.

\section{Introduction}

Hydrologic frequency analysis estimates occurrence probabilities of rare hydrologic events by fitting a probability distribution to observed extremes. Frequency analysis is used regularly in the design of hydrologic structures and in risk assessments, such as bridges, dams, power facilities, and floodplain and storm water management (Alila \& Mtiraoui, 2002; Bureau of Reclamation, 2003; Kumar et al., 2003). For example, during the late 1990s, the Bureau of Reclamation, which maintains over 300 dams located across 17 western states (Bureau of Reclamation, 2015), began transitioning to a risk-based approach to assess the safety of dams and prioritize expenditures. Hydrologic frequency analysis provides the necessary probabilistic information to conduct such dam safety evaluations and risk analyses (Bureau of Reclamation, 2003).

In the simplest form, frequency analysis can be conducted on a single variable at a single location. Quantiles from a fitted probability distribution give the magnitude of an event associated with a specified nonexceedance probability. In the case of annual maximum values, the return level (aka magnitude) associated with a $T$-year return period is the $(1-1 / T)$ th quantile of the distribution. A number of probability distributions are available to describe the behavior of extremes, including the generalized extreme value (GEV) distribution and the generalized pareto distribution (GPD), both of which are consistent with extreme value theory (EVT; Coles, 2001).

Given a daily time series of some variable, if the maximum value is selected from a predetermined block of time (annually, say), then the resulting time series will follow a three parameter GEV distribution 
(Westra et al., 2013), which can describe a wide range of tail behaviors. This approach is called the block maxima method. In the simplest form of block maxima frequency analysis, a stationary GEV distribution is fit to a data set at a single location, and return levels are computed for a range of return periods. A stationary GEV distribution is one in which the parameters of that distribution are constant in time.

Although the GEV distribution is flexible for modeling flood extremes, the Log Pearson type III (a Gamma distribution fitted to logarithm of the data) is widely used (Bobée, 1975; Bobee \& Robitaille, 1977) and is the legal standard in the United States. In this study, however, we focus our attention on the generalized extreme value (GEV) distribution due to its ability to capture a wide range of tail behaviors and its use in previous hydrologic studies (Davison et al., 2012; Ghosh \& Mallick, 2011; Katz, 2010; Katz et al., 2002; Najafi \& Moradkhani, 2014; Renard, 2011; Sang \& Gelfand, 2009).

There is growing evidence of the effects of climate change on hydroclimate extremes (Katz, 2010; Milly et al., 2008), highlighting the importance of nonstationary analyses. Under the assumption of stationarity, parameters of the GEV distribution are assumed to be constant in time, and consequently return levels are also constant in time. Nonstationary frequency analysis incorporates time varying covariates into the estimates of GEV parameters (Aryal et al., 2009; Cheng et al., 2014; Machado et al., 2015; Ouarda \& El Adlouni, 2011; Tan \& Gan, 2015). In a simple nonstationary case, a linear time trend can be incorporated in the GEV parameters (Cheng et al., 2014). Effects of large-scale climate can also be incorporated by including climate covariates such as the El Niño Southern Oscillation (ENSO) and the Pacific Decadal oscillation (PDO; Renard \& Lall, 2014).

Bayesian approaches to fitting GEV models provide several benefits beyond maximum likelihood estimation (MLE) methods. Frequency analyses are typically data limited, and MLE methods may diverge in these situations. In a Bayesian setting, through the use of priors, expert knowledge can be incorporated and model convergence can be improved (AghaKouchak et al., 2013). Priors represent assumed distributions assigned to each parameter that describes a given probability distribution. Finally, uncertainty is propagated naturally in a Bayesian model without the need for bootstrapping or other ad hoc uncertainty estimation methods (Cooley et al., 2007). Inability to converge or computational demands may restrict the use of these methods.

Regional frequency analysis (RFA) is a well-known procedure for incorporating observations at multiple locations in a homogeneous region where all the data are assumed to follow the same probability distribution (Bradley, 1998; Hosking \& Wallis, 1993). By including observations from multiple locations, RFA provides two main benefits over single-site models: (1) frequency estimates at ungauged locations and (2) improved estimates of distribution parameters at all the locations. Recent improvements to regional frequency analysis using a Bayesian hierarchical approach allow for estimation of streamflow quantiles at ungaged sites (Chebana et al., 2014; Lima et al., 2015; Yan \& Moradkhani, 2014), precipitation quantiles (Bracken et al., 2016; Renard, 2011), all with attendant uncertainties.

Multivariate frequency analysis improves upon regional frequency analysis by explicitly capturing the dependence between multiple hydrologic variables such as flow at two gages on a network, or two variables measured at the same location, say peak flow and volume. Dependence is typically captured by specifying a joint distribution for two or more variables. Favre et al. (2004) used a variety of copulas to capture the joint distribution of peak flows at two locations on a river network. Chebana and Ouarda (2011) specify a joint distribution for the quantiles of peak flow and volume using a copula. Yue and Rasmussen (2002), Kao and Govindaraju (2007), and Vandenberghe et al. (2011) conduct bivariate frequency analysis of rainfall and streamflow extremes using copulas. Another form of multivariate frequency analysis is the field of spatial extremes modeling (Bracken et al., 2016; Cooley et al., 2007).

Bayesian approaches have been proposed to conduct nonstationary frequency analysis. El Adlouni and Ouarda (2009) and Ouarda and El Adlouni (2011) conduct simultaneous model selection and parameter estimation of a nonstationary GEV model using a birth-death Monte Carlo Markov chain (MCMC) procedure and reversible jump MCMC, respectively. Both studies apply a Bayesian hierarchical modeling approach to model time varying parameters of the GEV distribution as a function of covariates and apply them to model extreme precipitation in California. The parameter estimation algorithm is different between the two. Steinschneider and Lall (2015) propose nonstationary regional frequency analysis conditioned on tropical moisture exports. O'Connell et al. (2002) conduct Bayesian flood frequency analysis which incorporates paleohydologic data and Renard (2011) proposes a fully Bayesian approach to regional frequency analysis. 
Multivariate frequency analysis using time varying copulas has been proposed to model nonstationarity. Jiang et al. (2015) apply a time varying copula with time and reservoir index as covariates to model flows at two locations on the Hanjiang River in China. Sarhadi et al. (2016a) proposed a Bayesian, dynamic conditional copula to model nonstationarity in multivariate drought attributes with discrete and continuous variables. They used this approach to propose a dynamic risk estimation framework for hydroclimate extremes under climate change (Sarhadi et al., 2016b). Kwon and Lall (2016) and Kwon et al. (2016) used copulas with a time varying model for distribution parameters of drought severity and duration over California and also applied them to paleo data to identify unprecedented nature of the recent drought from the joint return periods. Bender et al. (2014) employed time varying copulas with time varying extreme value distribution parameters to model flood peak and flood volume on the Rhine River.

Despite a large body of literature surrounding the topics of multivariate and nonstationary frequency analysis, a comprehensive Bayesian framework for conducting this analysis has yet to be proposed, which motivates this study. Here we develop such a framework using a Bayesian hierarchical approach. More specifically, this study brings together elements of several of the past studies, including the use of copulas, GEV marginal distributions, and nonstationary GEV parameters into a unified multivariate Bayesian framework. We demonstrate this framework with a joint frequency analysis model of annual peak snow water equivalent (SWE), annual peak flow, and annual peak reservoir elevation at Taylor Park dam in Colorado, USA. Futhermore, we compare results from the joint frequency analysis with results from more common frequency estimation techniques. Section 2 describes the framework in general. The application set up for Taylor Park reservoir, Colorado, USA is then described followed by the specific form of the model and fitting method in section 3 . Section 4 describes the results; conclusions and discussion of results are presented in section 5 .

\section{Proposed Framework}

The proposed multivariate nonstationary frequency analysis framework has three broad components-(i) the model structure, (ii) computation of the likelihood function and specification of priors, and (iii) estimation of nonstationary return levels.

\subsection{General Model Structure}

In general, we wish to conduct a joint frequency analysis of $n$ variables, $v_{1}, \ldots, v_{n}$ observed over $m$ years. For the model to apply, each variable must be an annual or seasonal maxima time series such that each variable follows a GEV distribution. The proposed general model structure for $n$ variables is

$$
\begin{gathered}
\left(v_{1}(t), \ldots, v_{n}(t)\right) \sim C_{g}(\Sigma ;\{\boldsymbol{\mu}(t), \boldsymbol{\sigma}(t), \xi(t)\}) \\
v_{i}(t) \sim G E V\left(\mu_{i}(t), \sigma_{i}(t), \xi_{i}(t)\right), \quad i=1, \ldots, n \\
\mu_{i}(t)=g_{\mu i}\left(\mathbf{x}_{i}(t)^{T}, \boldsymbol{\mu}(t), \boldsymbol{\sigma}(t), \xi(t)\right), \quad i=1, \ldots, n \\
\sigma_{i}(t)=g_{\sigma i}\left(\mathbf{x}_{i}(t)^{T}, \boldsymbol{\mu}(t), \boldsymbol{\sigma}(t), \xi(t)\right), \quad i=1, \ldots, n \\
\xi_{i}(t)=g_{\xi i}\left(\mathbf{x}_{i}(t)^{T}, \boldsymbol{\mu}(t), \boldsymbol{\sigma}(t), \xi(t)\right), \quad i=1, \ldots, n
\end{gathered}
$$

where $C_{g}$ is an $n$-dimensional Gaussian elliptical copula with dependence matrix $\Sigma, \mu_{i}(t), \sigma_{i}(t)$ and $\xi_{i}(t)$ are the GEV parameters (location, scale, and shape) for variable $i$ at time $t, \mu(t)=\left[\mu_{i}(t)\right]_{i=1}^{n}, \boldsymbol{\sigma}(t)=\left[\sigma_{i}(t)\right]_{i=1}^{n}$, $\xi(t)=\left[\xi_{i}(t)\right]_{i=1}^{n}$. The functions $g_{\mu i}(\cdot), g_{\sigma i}(\cdot)$, and $g_{\xi i}(\cdot)$ are (potentially nonlinear) functions of covariates $\mathbf{x}_{i}$ $(t)^{T}$ and parameters of other variables. These functions can be used to represent nonlinear dependence relationships between variables. In most cases, the $g .(\cdot)$ functions will be linear functions of covariates unless some specific nonlinear dependence structure is required.

The copula dependence matrix, $\Sigma$, is a symmetric positive definite matrix capturing the strength of dependence between each pairwise variable. The $i$, jth element of $\Sigma$ measures the dependence between variables $i$ and $j$ and can take values between -1 and 1 . By definition, the dependence between a variable and itself is unity so the diagonal elements of $\Sigma$ are 1 's 


$$
\Sigma=\left[\begin{array}{ccccc}
1 & v_{12} & \cdots & v_{1, n-1} & v_{1 n} \\
v_{12} & 1 & \ddots & & v_{2 n} \\
v_{13} & v_{23} & \ddots & & \vdots \\
\vdots & \vdots & \ddots & 1 & v_{n-1, n} \\
v_{1 n} & v_{2 n} & \cdots & v_{n-1, n} & 1
\end{array}\right]
$$

Note that since $\Sigma$ is symmetric, there are $n(n-1) / 2$ dependence parameters to fit (values in the lower or upper triangle of $\Sigma$ ). For a model with $n$ variables and $p$ covariates for each variable, this framework requires $n(p+3)+n(n-1) / 2$ parameters to be estimated. The Gaussian copula only assumes linear correlation after quantile transformation of the marginals with the inverse normal CDF. This does not impose a linear correlation structure on the marginal distributions, and in fact nonlinear dependence between variables can be captured at the data level.

A Gaussian elliptical copula is a specification for a multivariate distribution with predefined marginals (Renard \& Lang, 2007). Because of their ability to represent any marginal distribution, elliptical copulas have become a popular practical tool for modeling multivariate extremes (Ghosh \& Mallick, 2011; Renard, 2011; Renard \& Lang, 2007; Sang \& Gelfand, 2010).

Let $\mathbf{V}(t)=\left(V_{1}(t), \ldots, V_{n}(t)\right)$ be a random vector of hydrologic variables at time $t$ and let $\mathbf{v}(t)=\left(v_{1}(t), \ldots, v_{n}(t)\right)$ be a vector of the corresponding realizations. The Gaussian copula constructs the joint cumulative distribution function (cdf) of $\mathbf{V}(t)$ as

$$
F_{C}(\mathbf{v}(t))=\Phi_{\Sigma}(\mathbf{u}(t))
$$

where $\Phi_{\Sigma}(\cdot)$ is the joint cdf of an $n$-dimensional multivariate normal distribution with covariance matrix $\Sigma$, $\mathbf{u}(t)=\left(u_{1}(t), \ldots, u_{n}(t)\right), u_{i}(t)=\phi^{-1}\left(F_{i t}\left[v_{i}(t)\right]\right), \phi^{-1}$ is the inverse cdf (quantile function) of the standard normal distribution, and $F_{i t}(\cdot)$ is the marginal GEV cdf of variable $i$ at time $t$. The corresponding joint pdf is

$$
f_{C}(\mathbf{v}(t))=\frac{\prod_{i=1}^{n} f_{i}\left[v_{i}(t)\right]}{\prod_{i=1}^{n} \psi\left[u_{i}(t)\right]} \Psi_{\Sigma}(\mathbf{u}(t))
$$

where $f_{i t}(\cdot)$ is the marginal GEV pdf for variable $i$ at time $t, \psi(\cdot)$ is the standard normal pdf, and $\Psi_{\Sigma}(\cdot)$ is the joint pdf of an $n$-dimensional multivariate normal distribution.

\subsection{Likelihood and Priors}

Let $\mathbf{y}=\left[\mathbf{y}_{1}, \ldots, \mathbf{y}_{n}\right]$ be a matrix of observations where $\mathbf{y}_{i}=\left[y_{i}(t)\right]_{t=1}^{T}$ is a vector of observations at one site and $\mathbf{y}(t)=\left[y_{i}(t)\right]_{i=1}^{n}$ be a vector of all variables at time $t$. Let $\mathbf{x}_{i}=\left[x_{i}(t)\right]_{t=1}^{T}$ be a matrix of covariates for variable $i, X=\left[\mathbf{x}_{1}, \ldots, \mathbf{x}_{n}\right]$ be a matrix of all covariates and let $\boldsymbol{\theta}$ be a vector of latent variable parameters for the $g .(\cdot)$ functions. By Bayes' rule the posterior is

$$
p(\Sigma, \boldsymbol{\theta} \mid \mathbf{y}, \mathbf{X}) \propto p(\mathbf{y}, \mathbf{X} \mid \boldsymbol{\theta}) p(\Sigma, \boldsymbol{\theta})
$$

where the first term on the right-hand side is the copula likelihood which assuming temporal independence can be written

$$
p(\Sigma, \boldsymbol{\theta} \mid \mathbf{y}, \mathbf{X})=\prod_{t=1}^{m} p(\mathbf{y}(t), \mathbf{X} \mid \Sigma, \boldsymbol{\theta})
$$

where $p(\Sigma, \boldsymbol{\theta} \mid \mathbf{y}, \mathbf{X})$ is given by the likelihood in equation (8).

The marginal GEV likelihood, where temporal dependence is only considered for the location parameter, is

$$
f_{i}\left(y_{i}(t) \mid \mu_{i}(t), \sigma_{i}, \xi_{i}\right)=\frac{1}{\sigma_{i}} b_{i}^{-1 / \xi_{i}-1} \exp \left(-b_{i}^{-1 / \xi_{i}}\right)
$$

where 


$$
b_{i}=1+\xi_{i}\left(\frac{y_{i}(t)-\mu_{i}(t)}{\sigma_{i}}\right)
$$

In practice, the logarithm of the posterior is computed to prevent numerical underflow. In this case, the log likelihood is

$$
\begin{gathered}
\log p(\Sigma, \boldsymbol{\theta} \mid \mathbf{y}, \mathbf{X})=\log \prod_{i=1}^{n} p(\mathbf{y}(t), \mathbf{x} \mid \Sigma, \boldsymbol{\beta}, \boldsymbol{\sigma}, \boldsymbol{\xi}) \\
=\sum_{i=1}^{n} \log f_{i}\left[y_{i}\right]-\sum_{i=1}^{n} \log \psi\left[u_{i}\right]+\log \Psi_{\Sigma}\left(u_{1}, \ldots, u_{n}\right)
\end{gathered}
$$

where

$$
\sum_{i=1}^{n} \log f_{i}\left[y_{i}\right]=-\sum_{i=1}^{n}\left[\log \left(\sigma_{i}\right)+\left(1+1 / \xi_{i}\right) \log \left(b_{i}\right)+b_{i}^{-1 / \xi_{i}}\right]
$$

and

$$
b_{i}=1+\xi_{i}\left(y_{i}-\mu_{i}\right) / \sigma_{i}
$$

The term $p\left(\Sigma, \boldsymbol{\beta}_{\mu}, \boldsymbol{\sigma}, \boldsymbol{\xi}\right)$ from equation (9) contains prior information. Uninformative priors can be used for most parameters, except for the GEV shape parameters. Informative priors may be necessary to restrict GEV shape parameters to reasonable ranges for particular variables. For example, for precipitation, the shape parameter is usually less the 0.5 and positive so we could apply $p(\xi) \sim N(0,0.3)$ (Renard, 2011) or the so-called "geophysical prior" (a shifted beta distribution; Martins \& Stedinger, 2010). Priors such as these can help with model convergence and identifiability (AghaKouchak et al., 2013; Renard \& Lang, 2007).

\subsection{Nonstationary Return Levels}

Read and Vogel (2015) suggest it is important to be clear when discussing nonstationary return levels and return periods as there are several definitions (AghaKouchak et al., 2013; Cheng et al., 2014; Salas \& Obeysekera, 2014). The return level for a stationary GEV distribution is defined as the $p=(1-1 / T)$ th quantile

$$
q_{p}=\mu+\frac{\sigma}{\xi}\left[(-\log p)^{-\xi}-1\right]
$$

In a nonstationary setting when the GEV parameters may be time varying, the return level can be computed in each year, which is known as the effective return level (Cheng et al., 2014)

$$
q_{p}(t)=\mu(t)+\frac{\sigma(t)}{\xi(t)}\left[(-\log p)^{-\xi(t)}-1\right]
$$

In the case of nonstationary GEV parameters, a stationary return level can be computed based on quantiles of the nonstationary parameters

$$
\tilde{q_{p}}=\tilde{\mu}+\tilde{\sigma} \tilde{\xi}\left[(-\log p)^{-\tilde{\xi}}-1\right]
$$

where

$$
\begin{aligned}
\tilde{\mu} & =Q_{k}\left(\mu\left(t_{1}\right), \ldots, \mu\left(t_{m}\right)\right) \\
\tilde{\sigma} & =Q_{k}\left(\sigma\left(t_{1}\right), \ldots, \sigma\left(t_{m}\right)\right) \\
\tilde{\xi} & =Q_{k}\left(\xi\left(t_{1}\right), \ldots, \xi\left(t_{m}\right)\right)
\end{aligned}
$$

where $Q_{k}$ indicates computing the $k$ th quantile of the arguments which can be chosen based on the application. For example, choosing $k=0.5$ would represent the median behavior of each nonstationary GEV parameter. 


\section{Application}

In the Intermountain West, melting snowpack is the primary source of spring flood events at many highelevation watersheds (Hamlet \& Lettenmaier, 2007). However, frequency statistics of snow depth are not always utilized in dam safety flood frequency analyses, even in watersheds where annual maximum streamflow events are controlled by snowmelt. In this study, we demonstrate an application of the general multivariate, nonstationary frequency analysis presented in the previos section using annual maximum snow water equivalent (SWE), reservoir inflow, and water surface elevation (WSE) for the Taylor Park watershed and Taylor Park dam, Colorado, USA (Figure 1).

\subsection{Data}

Daily SWE observations were obtained from sites in the Global Historical Climatology Network (Menne et al., 2012) that are located within $320 \mathrm{~km}$ of the Taylor Park dam. Daily water surface elevation and naturalized inflow data at the Taylor Park reservoir were downloaded from the Bureau of Reclamation's Upper Colorado Region webpage at http://www.usbr.gov/rsvrWater/faces/rvrOSMP.xhtml. We required SWE stations have a minimum of $85 \%$ data coverage during the cool-season (October-July) for a minimum of 9 years in an effort to retain stations with sufficient wintertime observations. In addition, for a SWE station to be included, the annual maximum SWE time series had to be significantly correlated (at the $95 \%$ confidence level) with the annual maximum time series of inflow to Taylor Park reservoir. A regional average of peak SWE was used as an indicator of regional snow availability.

Our analysis is restricted to water years from 1981 to 2015 based on the availability of snow data. Annual maximum daily SWE, water surface elevation, and calculated inflow were computed for each water year.

\subsection{Model Structure for Taylor Park Watershed}

In this section, we give the structure of the model for the Taylor Park application, which is a special case of the model presented in section 2.1 wherein we can explicitly represent nonlinear dependence between variables.

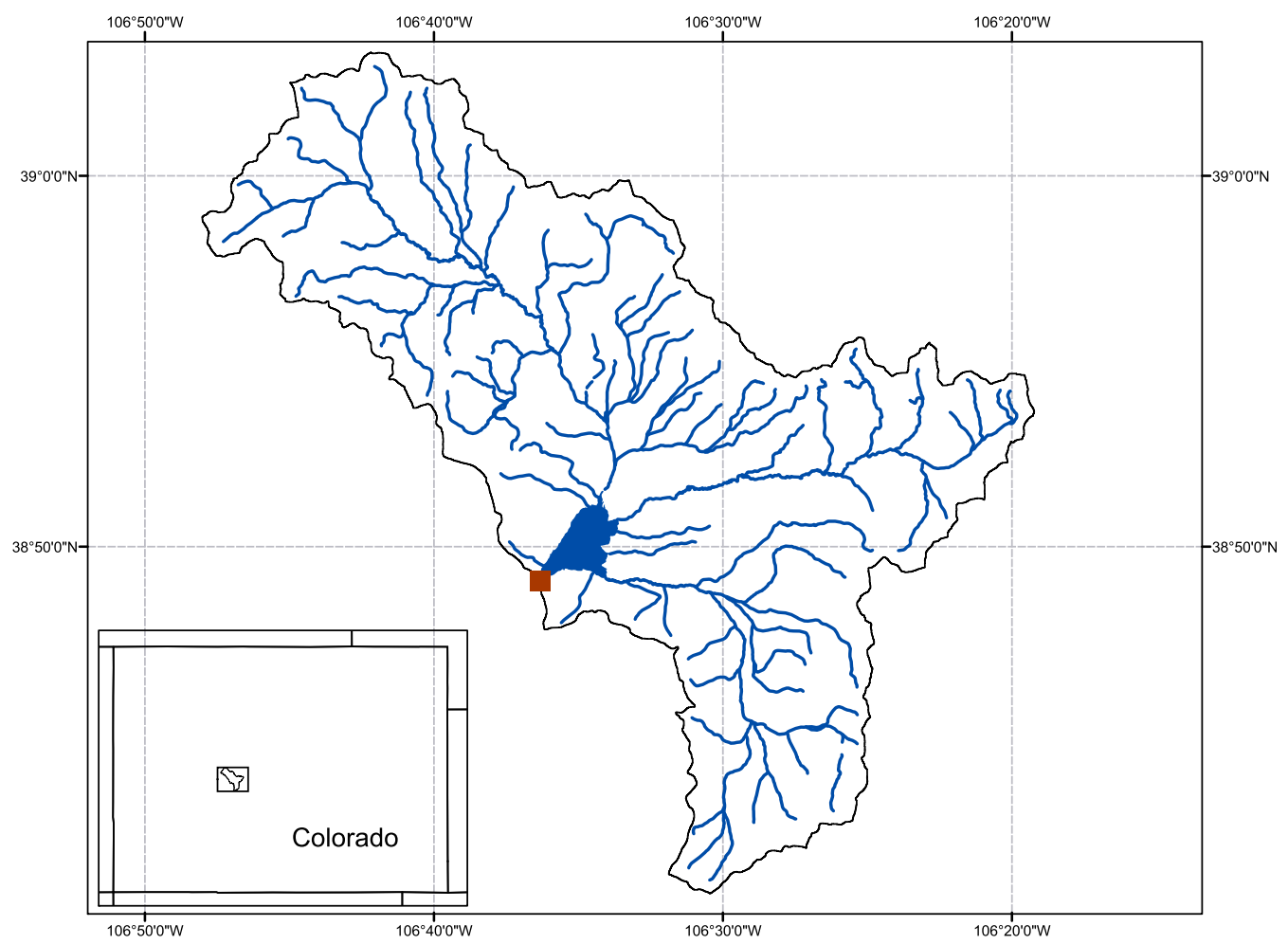

Figure 1. Outline of the Taylor Park watershed $\left(660 \mathrm{~km}^{2}\right.$; black line) located in Colorado, USA, with National Hydrologic Database stream locations (blue lines). Taylor Park dam is indicated by the brown square. 

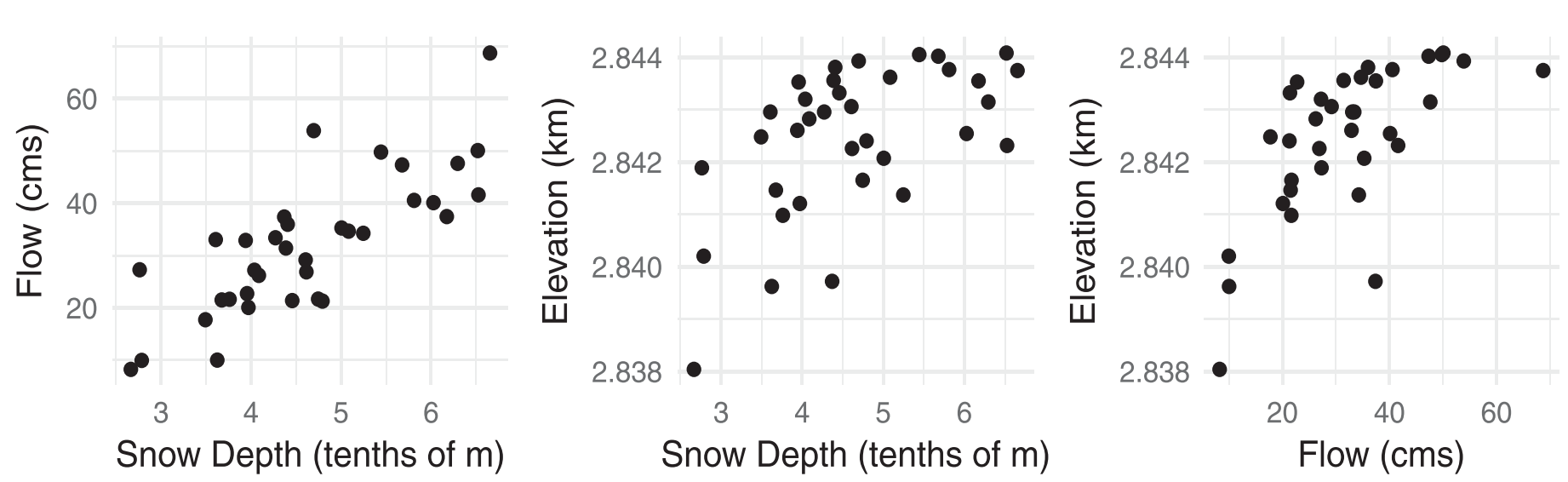

Figure 2. Pairwise scatterplots showing the dependence between peak SWE, peak flow, and peak elevation for Taylor Park dam.

Let $y(t)$ represent peak SWE, $z(t)$ represent peak flow, and $h(t)$ represent peak reservoir elevation. The model structure for these three variables is

$$
\begin{aligned}
&(y(t), z(t), h(t)) \sim C_{g}\left(\Sigma ;\left\{\mu_{y}(t), \sigma_{y}, \xi_{y}, \mu_{z}(t), \sigma_{z}, \xi_{z}, \mu_{h}(t), \sigma_{h}, \xi_{h}\right\}\right) \\
& y(t) \sim \operatorname{GEV}\left(\mu_{y}(t), \sigma_{y}, \xi_{y}\right) \\
& z(t) \sim \operatorname{GEV}\left(\mu_{z}(t), \sigma_{z}, \xi_{z}\right) \\
& h(t) \sim \operatorname{GEV}\left(\mu_{h}(t), \sigma_{h}, \xi_{h}\right) \\
& \mu_{y}(t)=\mu_{y 0}+\mathbf{x}_{y}(t)^{T} \boldsymbol{\beta}_{y} \\
& \mu_{z}(t)=\mu_{z 0}+\mathbf{x}_{z}(t)^{T} \boldsymbol{\beta}_{z} \\
& \mu_{h}(t)=\frac{a}{1+b c^{-\mu_{y}(t)}}
\end{aligned}
$$

where $\mathbf{x}_{y}(t)^{T}$ and $\mathbf{x}_{z}(t)^{T}$ are vectors of climate covariates for $y(t)$ and $z(t)$, respectively, $\mu_{y 0}$ and $\mu_{z 0}$ are variable specific intercept terms, and $\boldsymbol{\beta}_{y}$ and $\boldsymbol{\beta}_{z}$ are vectors of latent regression coefficients. In equation (29), we introduce a hierarchical nonlinear relationship between flow and reservoir elevation motivated by the nonlinear elevation relationships in Figure 2, right and middle, with parameter $a$ controlling the maximum reservoir elevation and $b$ and $c$ controlling the exact shape of the dependence relationship. Equation (29) demonstrates how we capture the nonlinear dependence between variables when it is supported by the data. For simplicity, we do not introduce time dependence for the scale and location parameters though this could be incorporated in a similar manner as the location parameter. In terms of the general model notation in section 2.2, $\boldsymbol{\theta}=\left[\mu_{y 0}, \boldsymbol{\beta}_{y}, \sigma_{y}, \xi_{y}, \mu_{z 0}, \boldsymbol{\beta}_{z}, \sigma_{z}, \xi_{z}, a, b, c\right]$ and $\mathbf{X}=\left[\mathbf{x}_{y}(t)^{T}, \mathbf{x}_{z}(t)^{T}\right]_{t=1}^{m}$.

The copula dependence matrix is

$$
\Sigma=\left[\begin{array}{ccc}
1 & v_{y z} & v_{y h} \\
v_{y z} & 1 & v_{z h} \\
v_{y h} & v_{z h} & 1
\end{array}\right]
$$

where $v_{i j}$ represents the dependence (correlation) between variable $i$ and $j$.

\subsection{Covariates}

We used indicies of the El Niño Southern Oscillation (ENSO), the Pacific Decadal Oscillation (PDO), and the Atlantic Multidecadal Oscillation (AMO) as covariates. We obtained values of the multivariate ENSO index (MEl; Wolter \& Timlin, 1998, 1993) from http://www.esrl.noaa.gov/psd/enso/mei/. PDO values (Zhang et al., 1997) were obtained from http://research.jisao.washington.edu/pdo/. AMO values (Enfield et al., 2001) were obtained from http://www.esrl.noaa.gov/psd/data/timeseries/AMO/. 
Peak SWE is expected to depend on winter values of each index so we used winter (December, January, and February; DJF) average values for covariates. Peak flow is expected to depend partially on snow (which is captured by the copula) and on spring values of each climate index, so we used spring (March, April, and May; MAM) average values as covariates. The covariates vectors at time $t$ are

$$
\begin{gathered}
\mathbf{x}_{y}(t)^{T}=\left[1, t, \operatorname{MEI}_{w}(t), \mathrm{PDO}_{w}(t), \mathrm{AMO}_{w}(t)\right] \\
\mathbf{x}_{z}(t)^{T}=\left[1, t, \operatorname{MEl}_{s}(t), \operatorname{PDO}_{s}(t), \mathrm{AMO}_{s}(t)\right]
\end{gathered}
$$

where a $w$ subscript denotes a winter average index and an $s$ subscript denotes a spring average index.

\subsection{Adequacy of the Gaussian Copula}

The Gaussian copula is a flexible tool for modeling multivariate distributions with prescribed marginal distributions (Renard \& Lang, 2007). Despite its flexibility, the Gaussian copula may not be appropriate for a given data set, that is, the data may not be multivariate normally distributed after transformation using $\phi^{-1}\left(F_{i}\left(y_{i}\right)\right)$ where $\phi^{-1}$ is the inverse cumulative standard normal distribution function and $F_{i}$ is the GEV distribution function for variable $y_{i}$. To test multivariate normality of the the data, we ran three tests, (1) Henze-Zirkler's (Henze \& Zirkler, 1990), (2) Mardia's (Mardia, 1970), and (3) Royston's (Royston, 1982) multivariate normality test using marginal transformations based on the MLE GEV fits. The $p$-values for the three tests were $0.56,0.73$, and 0.80 , respectively, all of which indicated the transformed data follow a multivariate normal distribution.

The Gaussian copula should be considered a practical tool for modeling multivariate extremes. However, its lack of asymptotic dependence may lead to underestimation of low probability events when used to extrapolate with data that exhibit tail dependence (Renard \& Lang, 2007). Renard and Lang (2007) suggest that the Gaussian copula is most useful in the observations range. In the results section, we show quantile curves extrapolated beyond the range of the data for the purposes of comparing uncertainty estimates.

\subsection{Model Fitting}

The model was fit using a Monte Carlo Markov Chain (MCMC) procedure, specifically, a univariate slice sampler (Neal, 2003) within a Gibbs sampler. In this scheme, slice sampling is applied to sample from the conditional distribution of each parameter given all other parameters. We ran three chains of length 100,000, discarding the first 50,000 iterations as burn-in and retaining only every 10 samples afterward, resulting in 15,000 posterior samples. To assess convergence, the $\hat{R}$ statistic (Gelman \& Rubin, 1992) was computed for each parameter, ensuring that the value was less than 1.1.

\subsection{Model Comparison}

We compare four related models to highlight the advantages of conducting a multivariate frequency analysis. The four models are as follows:

1. MLE S. A univariate stationary GEV distribution is fit independently to each variable using MLE.

2. MLE NS. A univariate nonstationary GEV distribution is fit to each variable using MLE where the mean is allowed to vary over time according to linear trend and climate covariates.

3. Bayes Ind. A univariate nonstationary GEV distribution is fit to each variable using MCMC where the mean is allowed to vary over time according to linear trend and climate covariates.

4. Bayes Joint. A multivariate Gaussian elliptical copula with nonstationary GEV marginals is fit to all variables simultaneously.

Uncertainty for the Bayesian methods is estimated naturally as part of the sampling procedure. To estimate uncertainty for the MLE method, we used the Hessian matrix evaluated at the posterior mode of the optimal parameter set, obtained from the maximum likelihood optimizer. From this matrix, we can estimate the covariance matrix of the parameters. Using this covariance matrix, we draw samples of parameters from a multivariate normal distribution (which is assumed by MLE). Each parameter sample drawn from the MVN (which determines a unique GEV distribution) was used to compute a return level shown in the paper.

\section{Results}

Figure 3 shows the nonstationary return levels for the 100 year return period from the Bayesianindependent model (left column), the Bayesian joint model (middle column), and the MLE-independent 


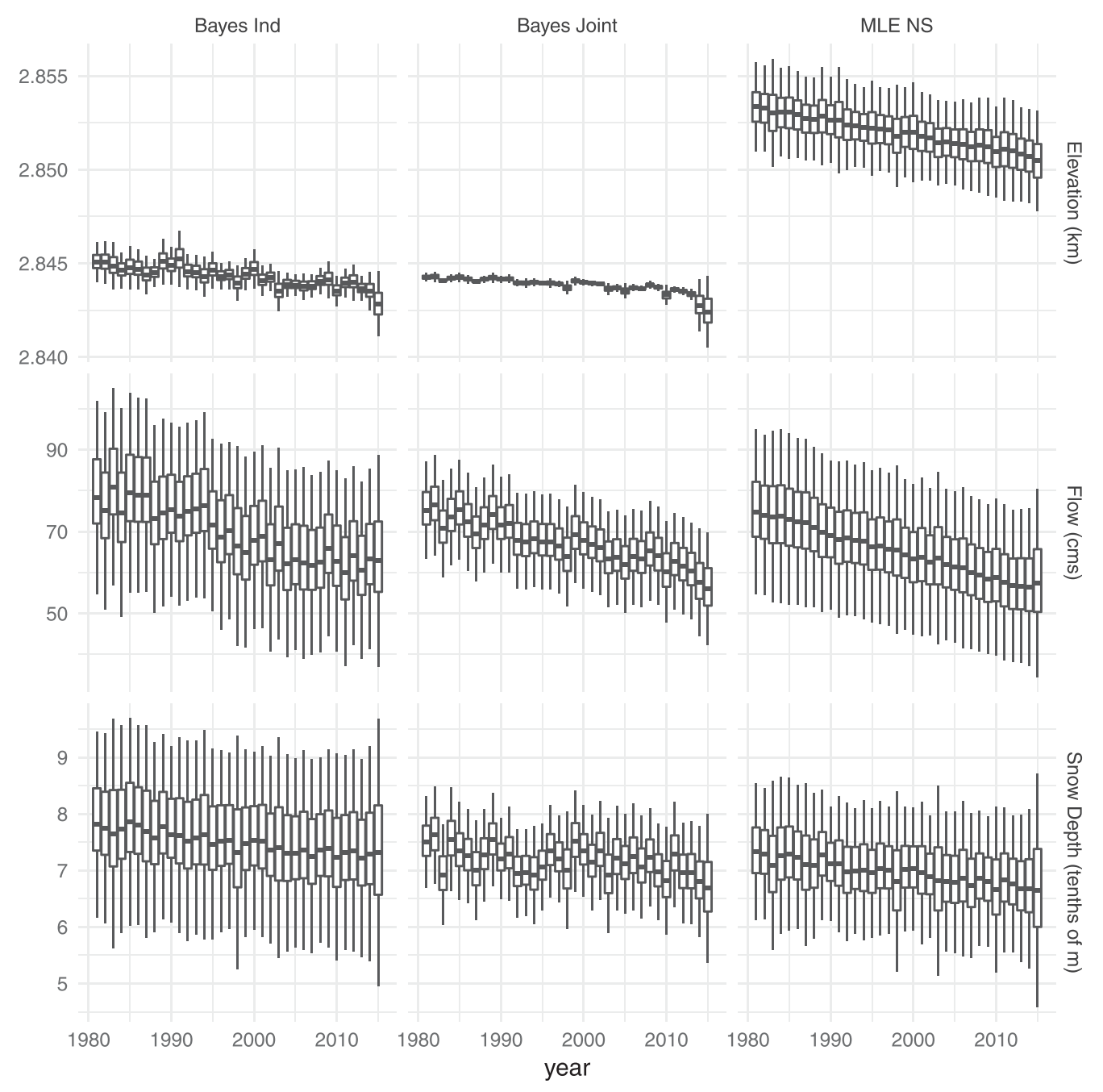

Figure 3. Nonstationary 100 year return levels from (left column) the independent Bayesian model, (middle column) the joint Bayesian model, and (right column) the independent MLE model. Reservoir elevation is shown in the top row, peak flow in the middle row, and peak SWE in the bottom row.

model for the three variables-reservoir elevation (top row), peak flow (middle row), and peak SWE (bottom row). The whiskers show the $90 \%$ credible intervals, the boxes the interquartile range, and the horizontal lines inside the boxes, the median. The reduction in uncertainty in the joint model compared to the independent models is apparent in all variables. Although all models use the same climate covariates, the MLEindependent model is less sensitive to the climate covariates. Both Bayesian models show similar trends but with increased uncertainty in the independent case. A downward linear trend is apparent in all variables and models. The top of the dam is $2848 \mathrm{~m}$ and the MLE-independent model consistently estimates higher reservoir water levels for the 100 year return (top right figure), which seems unrealistic, compared to the other two models. This is consistent with the model's insensitivity to climate covariates. In Figure 3, the joint model demonstrates increased year to year variability as compared to the MLE model. This is a distinct benefit of the joint model, by lending strength across variables the model is more flexible in its ability to capture year to year changes in the large-scale climate system, brought in through the covariates.

To compare the uncertainty estimates of all models, stationary return levels were computed from the nonstationary models using equation (19). That is, the fiftieth percentile of the nonstationary location parameter estimates were computed $(k=0.50)$ and used to compute stationary return levels for a range of return periods (1-1,000 year). Figure 4 shows the width of the $90 \%$ credible intervals for return levels plotted for a range of return periods. Smaller values of the width indicate lower uncertainty. The Bayesian joint model 


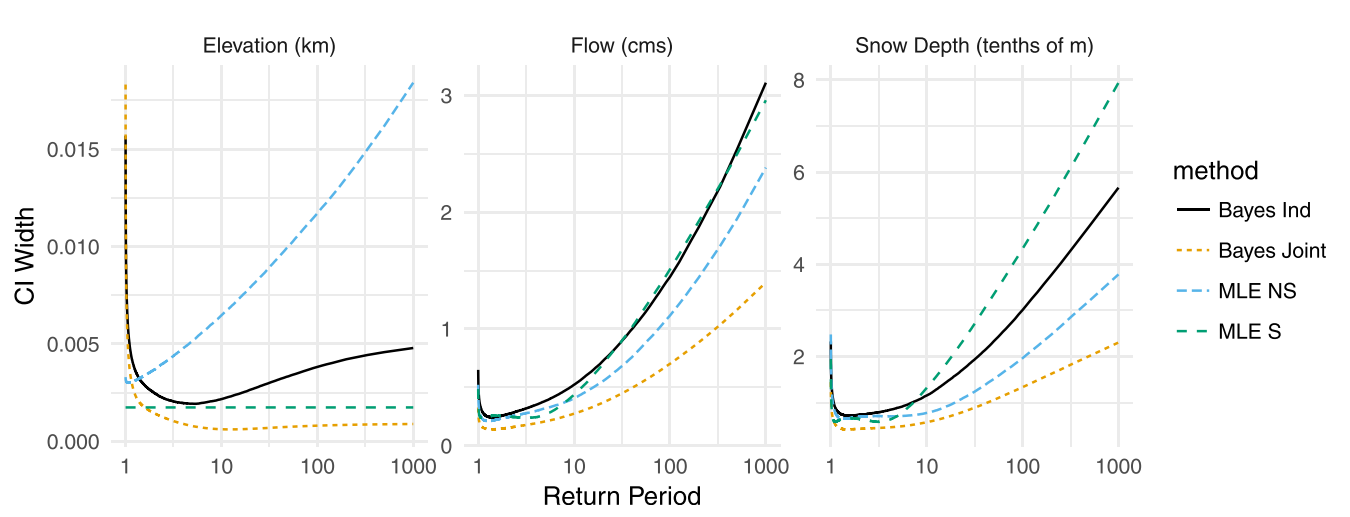

Figure 4. Width of the $90 \%$ credible intervals ( $\mathrm{Cl}$ ) of return levels for a range of return periods highlighting the reduction of uncertainty by conducting a joint frequency analysis.

produces lower uncertainty intervals for reservoir elevation flow, and snow depth compared to either the Bayesian-independent model or the MLE-independent model.

Uncertainty reduction is also apparent when inspecting posterior distributions of the parameters in common between the joint and independent Bayesian models (Figure 5). Posterior distributions for the joint model are less diffuse and in some cases demonstrate substantially different modes. This reduction of

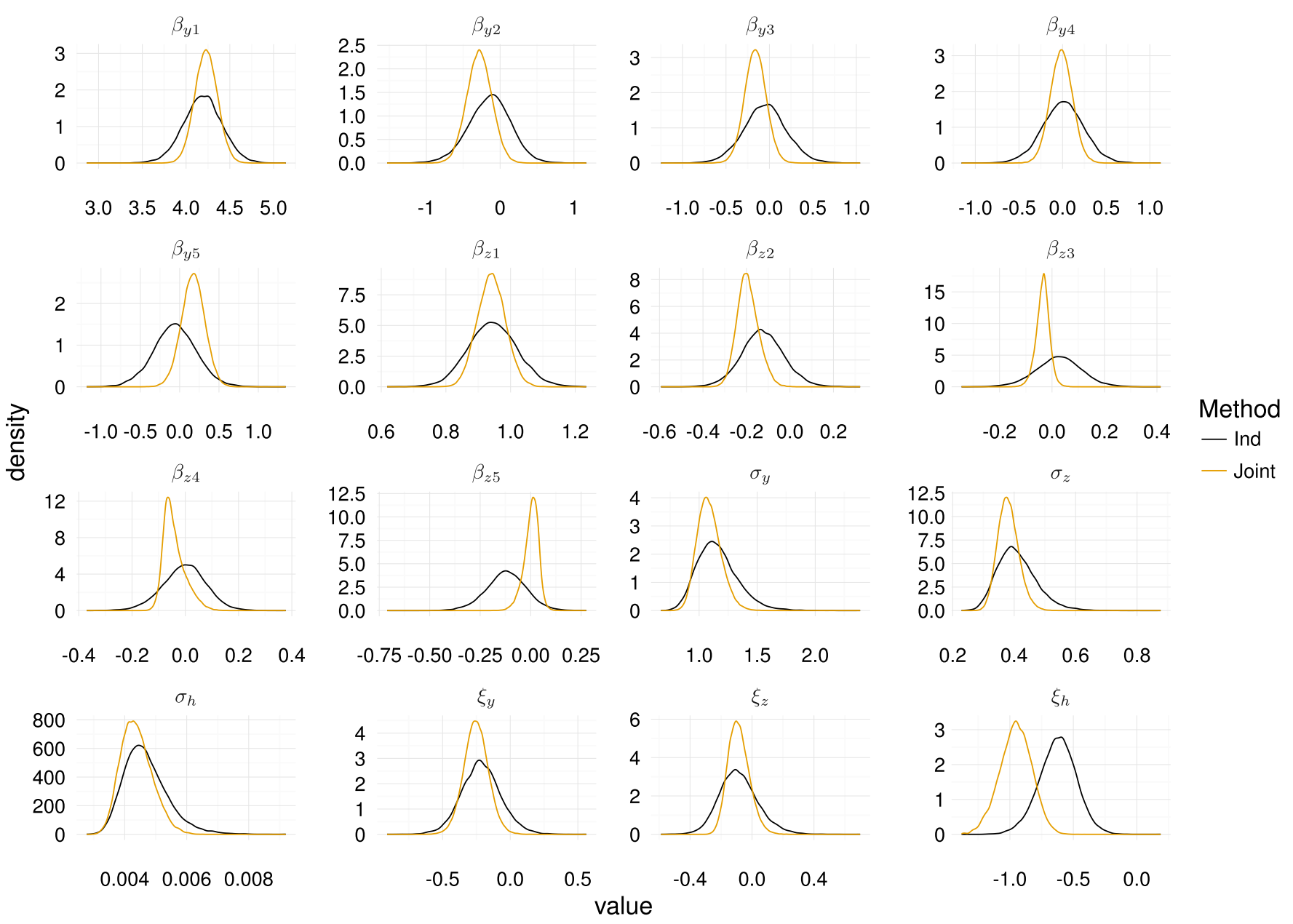

Figure 5. Posterior distributions of parameters in common between the joint and independent Bayesian models. 


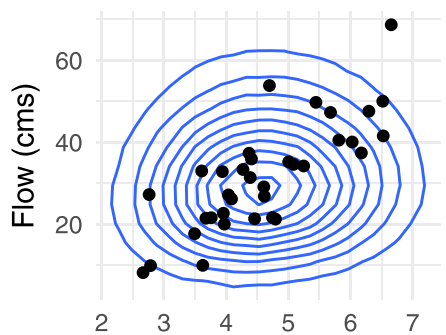

Snow Depth (tenths of $\mathrm{m}$ )

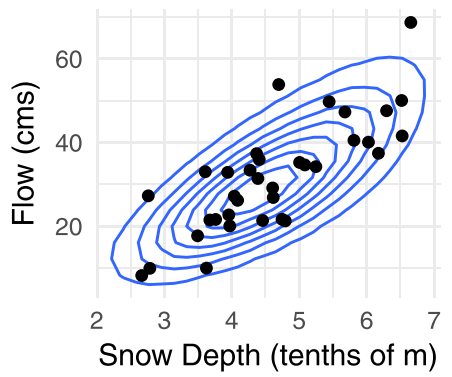

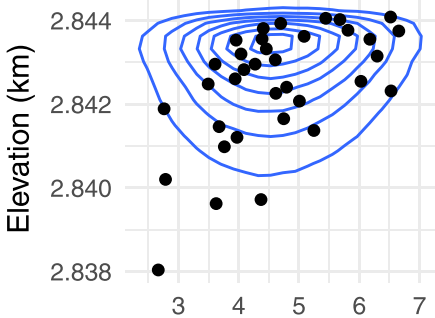

Snow Depth (tenths of $\mathrm{m}$ )

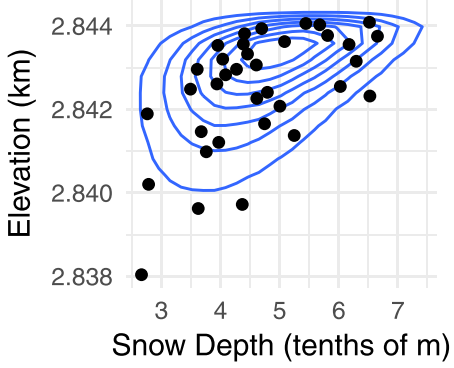

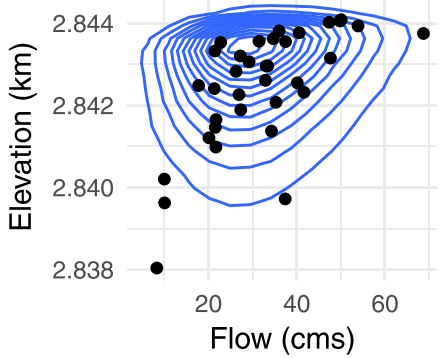

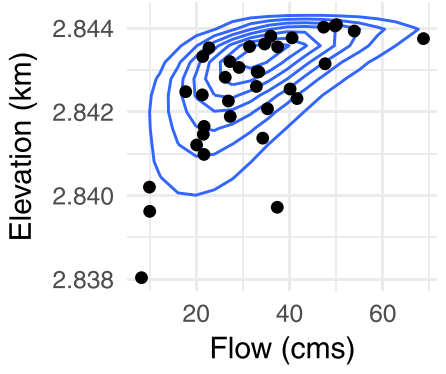

Figure 6. Pairwise dependence structure simulated (top row) without the copula and (bottom row) with the copula. Observations are shown as black points.

uncertainty in the posterior distributions is directly responsible for the reduction of uncertainty in the return level estimates.

The simulated model dependence structure between the variables using the Gaussian copula and without is compared with the observations (Figure 6 , bottom). It can be seen that the copula captures the structure of the pairwise dependence better (Figure 6, bottom) as compared to the simulations without the copula (Figure 6 , top). This property is important for simulating extremes while maintaining multivariate dependence.

\section{Conclusion and Discussion}

In this study, we presented a Bayesian hierarchical framework for modeling multivariate nonstationary frequency for hydrologic variables. The general framework assumes the marginal distribution of each variable follows a generalized extreme value (GEV) distribution, where the distribution parameters can vary in time as a function of covariates and of other model parameters. Multivariate dependence between variables is captured using a Gaussian elliptical copula.

We applied this framework to conduct a joint frequency analysis of peak snow water equivalent, peak inflow, and peak reservoir elevation for Taylor Park reservoir, Colorado, USA. This application incorporated large-scale climate covariates and a nonlinear relationship between flow and reservoir elevation. We found that the Bayesian approach provided robust estimation of uncertainties of the return levels and furthermore, use of the copula decreased uncertainty in return level estimates and captured the multivariate dependence between these three variables with high fidelity to the observed data.

The proposed Bayesian multivariate nonstationary framework has several benefits when compared to stationary, independent, and non-Bayesian versions:

1. Reduction of uncertainty. By incorporating multivariate dependence between variables, overall uncertainty was reduced.

2. Variable dependence. Linear dependence relationships between variables can be captured by using the copula.

3. Climate covariates. Covariates representing large-scale climate influences can be included to capture decadal and multidecadal climate oscillations.

4. Hierarchical dependence. Nonlinear dependence between variables (such as with flow and reservoir elevation) can be captured by incorporating nonlinear relationships between model parameters. 
The Gaussian copula is a flexible tool for modeling multivariate dependence between variables but may not always be appropriate in every application. For example, Renard and Lang (2007) give an example using peak flow and peak volume data from the Ubaye River at Barcelonette, France-wherein, peak flows are generated by either snowmelt or heavy rainfall and capturing this relationship with a single dependence parameter leads to poor results. It is therefore important to test for multivariate normality of a data set before a multivariate frequency analysis is conducted. The Gaussian copula also assumes asymptotic independence; in applications where tail dependence is present, extrapolating using the Gaussian copula may lead to underestimation of risk.

In the application we have presented, the scale and shape parameters were assumed to be stationary for simplicity. However, this assumption can be easily relaxed by adding covariates for these GEV parameters as well. While the proposed framework is focused on nonstationary analysis of three variables, with minimal effort this framework can be modified to include a spatial component to model multivariate extremes at several locations-such as on a river network, something we plan to investigate further.

\section{Acknowledgments}

Funding for this research was provided by a Science and Technology grant from Bureau of Reclamation, which is gratefully acknowledged. Thanks are due to three anonymous reviewers for their insightful comments which improved the manuscript. Preprocessing and postprocessing analysis was conducted using the $\mathrm{R}$ language (R Core Team, 2014). Data used can be found at http://civil. colorado.edu/bracken/multivariate extremes/data/.

\section{References}

AghaKouchak, A., Easterling, D., Hsu, K., Schubert, S., \& Sorooshian, S. (Eds.). (2013). Extremes in a changing climate: Detection, analysis and uncertainty. Berlin, Germany: Springer.

Alila, Y., \& Mtiraoui, A. (2002). Implications of heterogeneous flood-frequency distributions on traditional stream-discharge prediction techniques. Hydrological Processes, 16(5), 1065-1084.

Aryal, S. K., Bates, B. C., Campbell, E. P., Li, Y., Palmer, M. J., \& Viney, N. R. (2009). Characterizing and modeling temporal and spatial trends in rainfall extremes. Journal of Hydrometeorology, 10(1), 241-253.

Bender, J., Wahl, T., \& Jensen, J. (2014). Multivariate design in the presence of non-stationarity. Journal of Hydrology, 514, 123-130.

Bobée, B. (1975). The Log Pearson type 3 distribution and its application in hydrology. Water Resources Research, 11(5), $681-689$.

Bobee, B. B., \& Robitaille, R. (1977). The use of the Pearson type 3 and Log Pearson type 3 distributions revisited. Water Resources Research, 13(2), 427-443.

Bracken, C., Rajagopalan, B., Cheng, L., Kleiber, W., \& Gangopadhyay, S. (2016). Spatial Bayesian hierarchical modeling of precipitation extremes over a large domain. Water Resources Research, 52, 6643-6655. https://doi.org/10.1002/2016WR018768

Bradley, A. A. (1998). Regional frequency analysis methods for evaluating changes in hydrologic extremes. Water Resources Research, 34(4), 741-750.

Bureau of Reclamation. (2003). Probabilistic extreme flood hydrographs that use paleoflood data for dam safety applications (Tech. Rep. DSO03-03). Washington, DC: United States Bureau of Reclamation.

Bureau of Reclamation. (2015). About Us. Retrieved from http://www.usbr.gov/main/about/fact.html

Chebana, F., Charron, C., Ouarda, T. B. M. J., \& Martel, B. (2014). Regional frequency analysis at ungauged sites with the generalized additive model. Journal of Hydrometeorology, 15(6), 2418-2428.

Chebana, F., \& Ouarda, T. B. M. J. (2011). Multivariate quantiles in hydrological frequency analysis. Environmetrics, 22(1), 63-78.

Cheng, L., AghaKouchak, A., Gilleland, E., \& Katz, R. W. (2014). Non-stationary extreme value analysis in a changing climate. Climatic Change, 127(2), 353-369.

Coles, S. (2001). An introduction to statistical modeling of extreme values, Springer series in statistics. London, UK: Springer.

Cooley, D., Nychka, D., \& Naveau, P. (2007). Bayesian spatial modeling of extreme precipitation return levels. Journal of the American Statistical Association, 102, 824-840.

Davison, A. C., Padoan, S. A., \& Ribatet, M. (2012). Statistical modeling of spatial extremes. Statistical Science, 27(2), 161-186.

El Adlouni, S., \& Ouarda, T. B. M. J. (2009). Joint Bayesian model selection and parameter estimation of the generalized extreme value model with covariates using birth-death Markov chain Monte Carlo. Water Resources Research, 45, W06403. https://doi.org/10.1029/ 2007WR006427

Enfield, D. B., Mestas Nuñez, A. M., \& Trimble, P. J. (2001). The Atlantic Multidecadal Oscillation and its relation to rainfall and river flows in the continental U.S. Geophysical Research Letters, 28(10), 2077-2080.

Favre, A. C., El Adlouni, S., Perreault, L., Thiémonge, N., \& Bobée, B. (2004). Multivariate hydrological frequency analysis using copulas. Water Resources Research, 40, W01101. https://doi.org/10.1029/2003WR002456

Gelman, A., \& Rubin, D. B. (1992). Inference from iterative simulation using multiple sequences. Statistical Science, 7(4), 457-472.

Ghosh, S., \& Mallick, B. K. (2011). A hierarchical Bayesian spatio-temporal model for extreme precipitation events. Environmetrics, 22(2), $192-204$.

Hamlet, A. F., \& Lettenmaier, D. P. (2007). Effects of 20th century warming and climate variability on flood risk in the Western U.S. Water Resources Research, 43, W06427. https://doi.org/10.1029/2006WR005099

Henze, N., \& Zirkler, B. (1990). A class of invariant consistent tests for multivariate normality. Communications in Statistics-Theory and Methods, 19(10), 3595-3617.

Hosking, J. R. M., \& Wallis, J. R. (1993). Some statistics useful in regional frequency analysis. Water Resources Research, 29(2), $271-281$.

Jiang, C., Xiong, L., Xu, C.-Y., \& Guo, S. (2015). Bivariate frequency analysis of nonstationary low-flow series based on the time-varying copula. Hydrological Processes, 29(6), 1521-1534.

Kao, S.-C., \& Govindaraju, R. S. (2007). A bivariate frequency analysis of extreme rainfall with implications for design. Journal of Geophysical Research, 112, D13119. https://doi.org/10.1029/2007JD008522

Katz, R. W. (2010). Statistics of extremes in climate change. Climatic Change, 100(1), 71-76.

Katz, R. W., Parlange, M. B., \& Naveau, P. (2002). Statistics of extremes in hydrology. Advances in Water Resources, 25(8-12), 1287-1304.

Kumar, R., Chatterjee, C., Kumar, S., Lohani, A., \& Singh, R. (2003). Development of regional flood frequency relationships using L-moments for middle Ganga plains subzone 1(f) of India. Water Resources Management, 17(4), 243-257.

Kwon, H.-H., \& Lall, U. (2016). A copula-based nonstationary frequency analysis for the 2012-2015 drought in California. Water Resources Research, 52, 5662-5675. https://doi.org/10.1002/2016WR018959 
Kwon, H.-H., Lall, U., \& Kim, S.-J. (2016). The unusual 2013-2015 drought in South Korea in the context of a multicentury precipitation record: Inferences from a nonstationary, multivariate, Bayesian copula model. Geophysical Research Letters, 43, 8534-8544. https://doi. org/10.1002/2016GL070270

Lima, C. H. R., Lall, U., Troy, T. J., \& Devineni, N. (2015). A climate informed model for nonstationary flood risk prediction: Application to Negro River at Manaus, Amazonia. Journal of Hydrology, 522, 594-602.

Machado, M. J., Botero, B. A., López, J., Frances, F., Díez-Herrero, A., \& Benito, G. (2015). Flood frequency analysis of historical flood data under stationary and non-stationary modelling. Hydrology and Earth System Sciences, 19(6), 2561-2576.

Mardia, K. V. (1970). Measures of multivariate skewness and kurtosis with applications. Biometrika, 57(3), 519.

Martins, E. S., \& Stedinger, J. R. (2010). Generalized maximum-likelihood generalized extreme-value quantile estimators for hydrologic data. Water Resources Research, 36, 737-744. https://doi.org/10.1029/1999WR900330

Menne, M. J., Durre, I., Vose, R. S., Gleason, B. E., \& Houston, T. G. (2012). An overview of the Global Historical Climatology Network-daily database. Journal of Atmospheric and Oceanic Technology, 29(7), 897-910.

Milly, P. C. D., Betancourt, J., Falkenmark, M., Hirsch, R. M., Kundzewicz, Z. W., Lettenmaier, D. P., \& Stouffer, R. J. (2008). Stationarity is dead: Whither water management? Science, 319(5863), 573-574.

Najafi, M. R., \& Moradkhani, H. (2014). A hierarchical Bayesian approach for the analysis of climate change impact on runoff extremes. Hydrological Processes, 28(26), 6292-6308.

Neal, R. M. (2003). Slice sampling. The Annals of Statistics, 31(3), 705-767.

O'Connell, D. R. H., Ostenaa, D. A., Levish, D. R., \& Klinger, R. E. (2002). Bayesian flood frequency analysis with paleohydrologic bound data. Water Resources Research, 38(5). https://doi.org/10.1029/2000WR000028

Ouarda, T. B. M. J., \& El Adlouni, S. (2011). Bayesian nonstationary frequency analysis of hydrological variables. Journal of the American Water Resources Association, 47(3), 496-505.

R Core Team. (2014). R: A language and environment for statistical computing. Vienna, Austria: R Foundation for Statistical Computing

Read, L. K., \& Vogel, R. M. (2015). Reliability, return periods, and risk under nonstationarity. Water Resources Research, 51, $6381-6398$. https://doi.org/10.1002/2015WR017089

Renard, B. (2011). A Bayesian hierarchical approach to regional frequency analysis. Water Resources Research, 47, W11513. https://doi.org/ 10.1029/2010WR010089

Renard, B., \& Lall, U. (2014). Regional frequency analysis conditioned on large-scale atmospheric or oceanic fields. Water Resources Research, 50, 9536-9554. https://doi.org/10.1002/2014WR016277

Renard, B., \& Lang, M. (2007). Use of a Gaussian copula for multivariate extreme value analysis: Some case studies in hydrology. Advances in Water Resources, 30(4), 897-912.

Royston, J. P. (1982). An extension of Shapiro and Wilk's W test for normality to large samples. Applied Statistics, $31(2), 115$.

Salas, J. D., \& Obeysekera, J. (2014). Revisiting the concepts of return period and risk for nonstationary hydrologic extreme events, Journal of Hydrologic Engineering, 19(3), 554-568.

Sang, H., \& Gelfand, A. E. (2009). Hierarchical modeling for extreme values observed over space and time. Environmental and Ecological Statistics, 16(3), 407-426.

Sang, H., \& Gelfand, A. E. (2010). Continuous spatial process models for spatial extreme values. Journal of Agricultural, Biological, and Environmental Statistics, 15(1), 49-65.

Sarhadi, A., Ausín, M. C., \& Wiper, M. P. (2016b). A new time-varying concept of risk in a changing climate. Scientific Reports, 6, 35755.

Sarhadi, A., Burn, D. H., Concepción Ausín, M., \& Wiper, M. P. (2016a). Time-varying nonstationary multivariate risk analysis using a dynamic Bayesian copula. Water Resources Research, 52, 2327-2349. https://doi.org/10.1002/2015WR018525

Steinschneider, S., \& Lall, U. (2015). A hierarchical Bayesian regional model for nonstationary precipitation extremes in Northern California conditioned on tropical moisture exports. Water Resources Research, 51, 1472-1492. https://doi.org/10.1002/2014WR016664

Tan, X., \& Gan, T. Y. (2015). Nonstationary analysis of annual maximum streamflow of Canada. Journal of Climate, 28(5), 1788-1805.

Vandenberghe, S., Verhoest, N. E. C., Onof, C., \& De Baets, B. (2011). A comparative copula-based bivariate frequency analysis of observed and simulated storm events: A case study on Bartlett-Lewis modeled rainfall. Water Resources Research, 47, W07529. https://doi.org/10. 1029/2009WR008388

Westra, S., Alexander, L. V., \& Zwiers, F. W. (2013). Global increasing trends in annual maximum daily precipitation. Journal of Climate, 26(11), 3904-3918.

Wolter, K., \& Timlin, M. S. (1993). Monitoring ENSO in COADS with a seasonally adjusted principal component index. Paper presented at Proceedings of the 17 th Climate Diagnostics, Norman, OK.

Wolter, K., \& Timlin, M. S. (1998). Measuring the strength of ENSO events: How does 1997/98 rank? Weather, 53(9), 315-324.

Yan, H., \& Moradkhani, H. (2014). A regional Bayesian hierarchical model for flood frequency analysis. Stochastic Environmental Research and Risk Assessment, 29(3), 1019-1036.

Yue, S., \& Rasmussen, P. (2002). Bivariate frequency analysis: discussion of some useful concepts in hydrological application. Hydrological Processes, 16(14), 2881-2898.

Zhang, Y., Wallace, J. M., \& Battisti, D. S. (1997). ENSO-like interdecadal variability: 1900-93. Journal of Climate, 10(5), $1004-1020$. 